\title{
Die Verfassung der Republik Korea
}

Aus dem Koreanischen übersetzt und mit einer Einleitung versehen von HansPeter Bialas, Wiesbaden 1977, 35 S., 22,-DM

(Veröffentlichungen der Sejong-Bibliothek des Seminars für Orientalische Sprachen bei der Universität Bonn, Reihe A, Bd. 1)

Als Beilage zu Heft 3/1974 dieser Zeitschrift erschien eine englische Übersetzung der Verfassung Südkoreas von 1972 von Kim Youn-Soo mit einer etwa ebenso langen verfassungsgeschichtlichen Einleitung wie $\mathrm{zu}$ dem vorliegenden Buch. Es ist verwunderlich, daß der Bearbeiter des vorliegenden Bandes diese Einleitung und Ausgabe in seiner fünf Jahre später erschienenen Bibliographie mit keinem Wort erwähnt, obwohl er einen Aufsatz von Kim Youn-Soo über die vorige Verfassung von 1962 in Heft 1/1971 dieser Zeitschrift zitiert. Da beide Verfasser in ihren Einleitungen denselben Sachverhalt, die Verfassungsgeschichte Südkoreas von 1948-1972, behandeln, wäre es nützlich, beide Darstellungen zu vergleichen. Die autorisierte englische Übersetzung aus Korean Observer, die, außer im oben erwähnten Beiheft, auch in der bekanntesten Verfassungssammlung der Welt von Blaustein/Flanz 1974 herausgegeben wurde, dort mit einer ausführlichen verfassungsgeschichtlichen Chronology (16 Seiten) und Bibliographie, ist leider auch nicht erwähnt. Es ist bedauerlich, daß die wissenschaftliche Zusammenarbeit so wenig koordiniert ist. Die deutsche Übersetzung als solche ist klar und verständlich, wird allerdings nur durch 26 knappe Fußnoten erläutert. So wäre z. B. bei Art. 2 ein Hinweis sinnvoll gewesen, daß in Südkorea immer noch das Staatsangehörigkeitsgesetz von 1948 (mit geringen Anderungen v. 1962 und 1963) gilt, das in Bd. 32, S. 279, der "Sammlung geltender Staatsangehörigkeitsgesetze“ (1971), in deutscher Übersetzung abgedruckt ist.

Dr. Hellmuth Hecker

\section{Peter A. Oluyede}

\section{Administrative Law in East Africa}

Nairobi - Kampala - Dar es Salaam 1973, XXI, 255 S.

Kenia, Uganda und Tansania, die ehemals Teile des britischen Kolonialreichs waren bzw. von ihm verwaltet wurden, sind heute drei politisch gegensätzliche Nachbarn in Ostafrika. Der nigerianische Verfasser des angezeigten Lehrbuchs, der selbst Verwaltungsrecht an der Universität von Dar es Salaam lehrt, macht den Versuch, das Verwaltungsrecht dieser drei Länder parallel darzustellen. Die Grundsätze und Rechtsbehelfe des englischen Verwaltungsrechts, dessen eigene Entwicklung noch keineswegs abgeschlossen ist, gelten nach seiner Auffassung im wesentlichen nach wie vor in den drei Staaten trotz aller grundlegenden Veränderungen ihrer politischen Verfassung seit der Unabhängigkeit. Eine Emanzipation von den Rechtsformen der früheren Kolonialmacht ist allerdings im Recht der öffentlichen Unternehmen $\mathrm{zu}$ beobachten, das im 6. Kapitel ausführlich diskutiert wird; dieses Rechtsgebiet hat heute etwa in Tansania weitaus größere Bedeutung als in der britischen "mixed economy“. Das lebendig geschriebene Buch kann zwar keine Auskunft über die Rechtswirklichkeit im heutigen Ostafrika geben, enthält aber dafür interessantes Material wie etwa den Briefwechsel Obote - Obwangor von 1967 (S. 17 ff.) und im Anhang den tansanischen Permanent Commission of Enquiry Act 1966.

Alexander Dix 